werden, für die bisher nur der Postweg infrage kam. Das De-MailGesetz, das am 3. Mai 2011 in Kraft trat, sorgt dafür, dass alle DeMail-Anbieter nach den gleichen Kriterien in einem transparenten Verfahren geprüft werden. Dadurch wird De-Mail deutschlandweit von allen De-Mail-Anbietern auf einem einheitlichen und geprüften Sicherheitsniveau angeboten.

\section{Allianz für Cyber-Sicherheit}

Zur Umsetzung der Cyber-Sicherheitsstrategie für Deutschland initiieren das Bundesamt für Sicherheit in der Informationstechnik (BSI) und der Bundesverband Informationswirtschaft, Telekommunikation und neue Medien e.V. (BITKOM) gemeinsam die "Allianz für Cyber-Sicherheit“. Ziel der im Rahmen der CeBIT 2012 angekündigten Allianz ist, zusammen mit weiteren Partnern aus Wirtschaft, Wissenschaft und Verwaltung die Cyber-Sicherheit in Deutschland weiter zu verbessern und aktiv zu gestalten. BSI und BITKOM laden daher alle Akteure in Deutschland, die sich mit Cyber-Sicherheit beschäftigen, ein, in der Allianz mitzuwirken. Insbesondere angesprochen sind dabei Hersteller, IT- und Telekommunikationsdienstleister, Träger der Internetinfrastrukturen, CERTs (Computer Emergency Response Teams), Anwenderbranchen mit intensivem IT-Einsatz sowie Multiplikatoren aus Medien und Wissenschaft.

Ausgehend von einem konsolidierten, aktuellen Lagebild werden im Rahmen der Allianz künftig Verwaltung, Wirtschaft und Wissenschaft mit Informationen und Lösungshinweisen zur Realisierung eines angemessenen Cyber-Sicherheitsniveaus versorgt. Dazu zählen eine größere Transparenz über aktuelle Angriffsformen und Schwachstellen in relevanten IT-Produkten sowie Empfehlungen zu notwendigen Sicherheitsmaßnahmen. Wesentlicher Bestandteil in dieser Kooperation ist zudem der Informations- und Erfahrungsaustausch zwischen Staat, Wirtschaft und Wissenschaft. Ein vertrauensvoller Austausch zum Thema Cyber-Sicherheit soll bewusst auch auf regionaler Ebene gestärkt werden, um Landesbetriebe, kommunale Dienstleister und mittelständische Unternehmen besser mit relevanten Informationen unterstützen zu können. Das BSI wird in der Allianz eine koordinierende Rolle einnehmen und - mit Zuarbeit aus dem Nationalen Cyber-Abwehrzentrum Cyber-Sicherheitsempfehlungen, Warnungen, Analysen sowie vertrauliche Hintergrundinformationen einbringen. Darüber hinaus übernimmt das BSI die Beobachtung der Gesamtlage sowie die nationale IT-Krisenreaktion.

BITKOM wird die Erkenntnisse zu Gefährdungs- und Angriffslage an die Hersteller und Dienstleister im Bereich Cyber-Sicherheit transportieren, damit zukünftig adäquate Lösungen bereitgestellt werden können. Gleichzeitig soll das Know-how der häufig weltweit tätigen Mitgliedsunternehmen in das aktuelle Lagebild einfließen. Zudem wird der BITKOM den Erfahrungsaustausch mit Großunternehmen, Vertretern von Anwenderbranchen und regionalen Unternehmerverbänden initiieren und intensivieren sowie mit dem BSI den Transfer der Informationen an die Zielgruppen übernehmen.

Aktuelle Gefährdungslage erfordert gemeinsames Handeln Praktisch alle Lebensbereiche des gesellschaftlichen Handelns, die Arbeitsfähigkeit der Verwaltung und die Wertschöpfung der Wirtschaft hängen von funktionierenden IT- und Internetstrukturen ab. Daher ist die zunehmende Zahl an Angriffen, die über den CyberRaum auf digitale Identitäten, vertrauliche Daten und die Verfüg- barkeit elektronischer Prozesse zielen, besorgniserregend. Technisch versierte Angreifer nutzen das Internet für kriminelle Aktivitäten, um die Handlungsfähigkeit von Verwaltung und Unternehmen einzuschränken oder um finanzielle Ziele zu erreichen: Identitätsdiebstahl, Verfügbarkeitsangriffe, Online-Spionage und -Sabotage sind tägliche Phänomene geworden. Szenarien, die vor Kurzem noch reine Theorie oder Fiktion waren, werden heute im CyberRaum von der Wirklichkeit überholt. Diese internationale Gefährdungssituation trifft auch Deutschland. Permanent finden CyberAttacken statt, die die Leistungsfähigkeit des Standortes Deutschland massiv beeinträchtigen können. Um dieser Entwicklung gemeinsam entgegenzuwirken, ist die intensive Kooperation von Staat und Wirtschaft erforderlich. Mit der Allianz für Cyber-Sicherheit wird hierfür die geeignete Plattform geschaffen.

\section{BSI: Leitfaden für eine Sichere IPv6- Netzwerkarchitektur}

Das Bundesamt für Sicherheit in der Informationstechnik (BSI) hat im Rahmen der Reihe: BSI-Standard zur Internet-Sicherheit (ISi-Reihe) am 05.03.2012 den Leitfaden für eine Sichere IPv6-Netzwerkarchitektur veröffentlicht.

Der Leitfaden richtet sich primär an Führungskräfte und IT-Koordinatoren. Er gibt Hinweise für die Vorgehensweise bei der Einführung des neuen Internetprotokolls IPv6 und zeigt Wege auf, wie die neuen Möglichkeiten von IPv6 sinnvoll genutzt werden können, um die Sicherheit des internen Netzes bei der Einführung von IPv6 durch eine angepasste Architektur zu gewährleisten.

Weitere Informationen auf https://www.bsi.bund.de/DE/Themen/ Cyber-Sicherheit/ISi-Reihe/ISi-LANA/lana_node.htmI

\section{Migrationsleitfaden in der Version 4.0 veröffentlicht}

Der Migrationsleitfaden (Leitfaden für die Migration von Software) steht ab sofort in der neuen Version 4.0 zum Download zur Verfügung.

Der Migrationsleitfaden untersucht rechtliche, wirtschaftliche als auch strategische und technische Aspekte bei Software-Migrationen, die in einem jeweils eigenen Dokument beleuchtet werden. Er enthält nunmehr vor allen Dingen Entscheidungshilfen für die jeweiligen Migrationsgebiete in Form von Kriterienlisten, kurzen Produktbeschreibungen, tabellarischen Gegenüberstellungen und Empfehlungen. Ergänzt werden diese um Begriffsdefinitionen, allgemeine Kriterien für erfolgreiche Software-Migrationen sowie einem Ausblick auf Zukunftsthemen der IT, aber auch rechtliche Fragen wie die Regelung der Haftung, Lizenzfragen oder auch der Umgang mit open Source im Vergabeverfahren.

Nach einer 8-wöchigen Konsultationsphase auf der Webseite des Kompetenzzentrums Open Source Software (CC-OSS) wurde der Migrationsleitfaden im Auftrag der Beauftragten der Bundesregierung für Informationstechnik abschließend überarbeitet und liegt nun unter http://www.cio.bund.de/mIf zum Download bereit. 